\title{
Circuit
}

Musiques contemporaines

\section{Concours international de composition du Festival Présences à Shanghai (2008-2010) : le moment où le Québec a rayonné en Chine par sa création musicale Presences Festival International Composition Competition in Shanghai (2008-2010): The Moment When Quebec Shined in China Through Music Creation}

\author{
Jessica Pilon Pinette et Danick Trottier
}

Volume 27, numéro 3, 2017

Illusions polyphoniques : José Evangelista et l’hétérophonie

URI : https://id.erudit.org/iderudit/1042839ar

DOI : https://doi.org/10.7202/1042839ar

Aller au sommaire du numéro

Éditeur(s)

Circuit, musiques contemporaines

ISSN

1183-1693 (imprimé)

1488-9692 (numérique)

Découvrir la revue

Citer cet article

Pilon Pinette, J. \& Trottier, D. (2017). Concours international de composition du Festival Présences à Shanghai (2008-2010) : le moment où le Québec a rayonné en Chine par sa création musicale. Circuit, 27(3), 49-61.

https://doi.org/10.7202/1042839ar
Résumé de l'article

C'est à la suite d'une immersion en Chine en 2008 ainsi qu'après avoir franchi les nombreuses étapes préliminaires de la grande finale internationale, que Denis Gougeon est déclaré en mai 2010 grand gagnant du Concours international de composition du Festival Présences à Shanghai. Présélectionnés par la SMCQ, huit compositrices et compositeurs québécois ont été invités à se familiariser avec la culture et la tradition musicale shanghaiennes en vue de participer à ce concours. La finale québécoise a ainsi pris place en 2009, moment où tous devaient présenter une composition intégrant un instrument et une mélodie issus de la tradition chinoise. Les oeuvres créées à cette occasion par Simon Bertrand, José Evangelista, Denis Gougeon, Analía Llugdar, Pierre Michaud, Farangis Nurulla-Khoja, Sean Pepperall et Serge Provost, ont offert une vitrine de choix pour la diffusion de la création musicale du Québec à l'international. L'enquête donne un aperçu des différentes étapes ayant marqué ce concours par l'intermédiaire des souvenirs et des propos de Simon Bertrand, Walter Boudreau, José Evangelista, Denis Gougeon, Analía Llugdar et Pierre Michaud, passant autant par l'organisation de la participation québécoise que par la manière dont chacun a vécu cette expérience et a conçu son oeuvre. 


\title{
Enquête
}

\section{Concours international de composition du Festival Présences à Shanghai (2008-2010): le moment où le Québec a rayonné en Chine par sa création musicale}

\author{
Jessica Pilon Pinette et Danick Trotter
}

C'est lors de la grande finale du Concours international de composition du Festival Présences à Shanghai, en 2010, que tous les regards se sont rivés sur le talent québécois en matière de composition musicale. Denis Gougeon a alors remporté le premier prix du concours avec Toy (Music Box) le 4 mai 2010, création assurée par l'Orchestre philharmonique de Radio-France au Grand Théâtre (Opéra) de Shanghai, sous la direction de François-Xavier Roth. C'est aux côtés du compositeur français Krystof Maratka ( $2^{\mathrm{e}}$ prix) et du compositeur danois Simon Steen-Andersen ( $3^{\mathrm{e}}$ prix) qu'il a concouru et a mérité cette victoire. Mais avant d'en arriver à ce point culminant du concours, de nombreuses étapes ont dû être franchies, s'inscrivant elles-mêmes dans le cadre d'évènements culturels majeurs, tels que le Festival international de musique Printemps de Shanghai, le Festival Présences, organisé par RadioFrance et René Bosc, ou encore l'Exposition universelle de 2010 à Shanghai.

Fruit d'un grand partenariat entre Radio-France et le Shanghai Media Group, en plus de la Société de musique contemporaine du Québec (SMCQ) pour la présence québécoise, ce concours de composition s'est échelonné sur plusieurs années. Ainsi, en 2006, la ville de Shanghai accueillait ses premiers participants pour la première étape du concours. Puis, en 2007, avait lieu la finale française, suivie, en 2008, de la danoise.

C'est au cours de cette même année que huit compositrices et compositeurs d'ici ont pris la voie des airs pour accomplir à leur tour la première étape $d u$ concours. Sélectionnés par Walter Boudreau, directeur artistique de la SMcQ, José Evangelista, Denis Gougeon, Serge Provost, Sean Pepperall, Simon Bertrand, Pierre Michaud, Analía Llugdar et Farangis Nurulla-Khoja ont passé dix jours dans la ville chinoise en mai 2008, se familiarisant ainsi avec la culture shanghaienne tout en rencontrant des musicologues spécialistes de la musique chinoise et en assistant à des performances d'interprètes professionnels de divers instruments traditionnels ${ }^{1}$.

1. L'année suivante, plus précisément le 11 mai 2009 dans le cadre du Shanghai Spring International Music Festival, leurs œuvres ont été créées au Grand Théâtre de Shanghai par l'Orchestre symphonique de Shanghai sous la direction de Zhang Yi. 
Mais pour l'un d'entre eux, plus particulièrement, cette expérience artistique orientée vers la culture musicale de l'Autre est une chose un peu plus familière. En effet, le parcours musical ainsi que l'œuvre de José Evangelista sont réputés pour leurs recours à des couleurs musicales dites ethniques ou traditionnelles, donc provenant de cultures musicales extérieures à l'Occident. Que ce soit ses racines hispaniques ou bien sa fidèle passion pour le gamelan, auxquelles s'est ajoutée dans le cadre de ce concours l'expérience d'une immersion dans la musique traditionnelle chinoise, les sources d'inspiration sont nombreuses chez ce compositeur.

Bref, le but était de favoriser une immersion afin que chacun puisse s'enrichir, autant personnellement qu'artistiquement. L'année suivante, en 2009, tous devaient présenter une œuvre d'une durée de cinq minutes. Tout comme pour les Français et les Danois, le compositeur québécois retenu devait ensuite transformer sa création en une œuvre plus substantielle. Les critères à respecter reposaient sur l'alliage des instruments traditionnels chinois et de l'orchestre occidental ainsi que sur l'intégration d'une mélodie folklorique chinoise. C'est lors de la finale québécoise de 2009 que Denis Gougeon a été sélectionné en vue de la grande finale internationale du concours, grâce à sa pièce Toy \#1/Proeludium Mechanicus et dont Toy (Music Box), œuvre gagnante de la grande finale internationale, est le prolongement; Sean Pepperall s'est vu accorder le second prix pour Happy Green Tea-Quake's Toll Resilience, tandis que José Evangelista a obtenu le troisième prix avec $O$ Qin $^{2}$. Cette

2. Notez qu'il est possible d'écouter toutes les compositions créées lors de cette finale québécoise sur la chaîne du Centre de musique canadienne (CMc), sur YouTube: www.youtube. com/user/cmcquebec/search?query=en+chine (consulté le 11 septembre 2017).
FIGURE 1 Première page d'O Qin de José Evangelista.

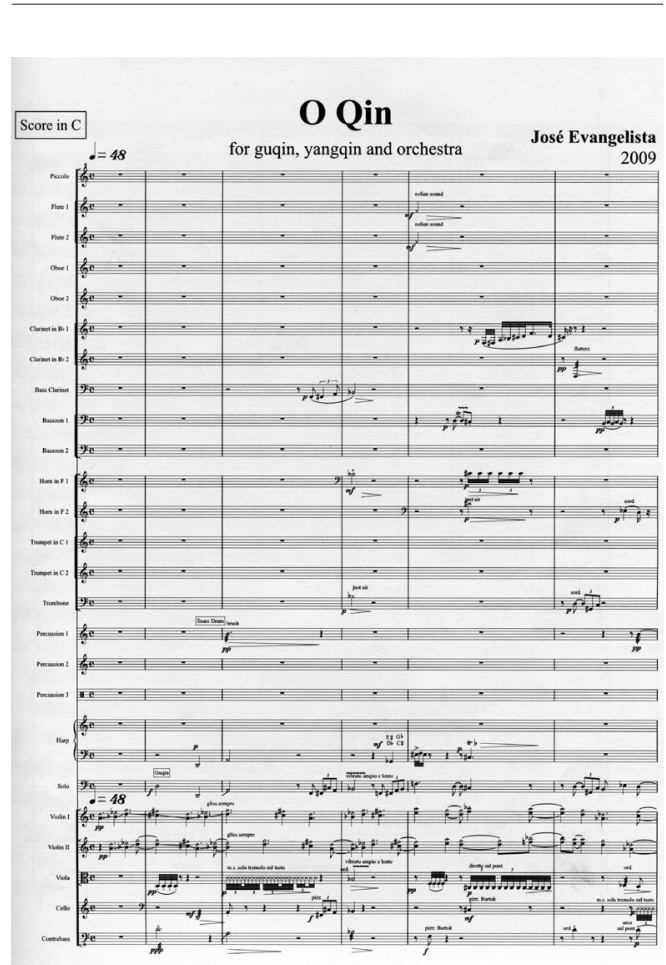

dernière met mélodiquement de l'avant les sonorités du guqin et du yangqin ${ }^{3}$ (voir Figure 1).

Plus d'une dizaine d'années après les débuts de ce concours, les compositrices et compositeurs, ainsi que les organisateurs de la SMCQ, en gardent toujours un vif souvenir. Parmi les huit participants, cinq d'entre eux aux côtés de Walter Boudreau ont bien voulu se prêter au jeu de nous faire revivre les moments clés de leur

3. Dans les deux cas, il s'agit d'instruments à cordes pincées qui s'apparentent à la famille des cithares. 
aventure musicale par les questions qui leur ont été adressées - certaines des réponses ont été transmises par courriels et d'autres recueillies lors d'entretiens au courant des mois de juin et de juillet 2017. C'est ainsi que les questions font état des diverses parties du concours vues sous l'angle de ses principaux artisans, l'enquête ayant pour objectif de résumer les principales étapes du concours. Nous tenons ainsi à remercier sincèrement et chaleureusement Walter Boudreau ${ }^{4}$, José Evangelista $^{5}$, Denis Gougeon ${ }^{6}$, Simon Bertrand ${ }^{7}$, Pierre Michaud $^{8}$ et Analía Llugdar ${ }^{9}$ d'avoir accepté de raviver leurs souvenirs en témoignant de l'expérience acquise au Concours international de composition à Shanghai. Nous tenons aussi à remercier grandement la SMCQ pour sa précieuse collaboration tout au long du développement de cette enquête.

\section{Prélude}

Comment la SMCQ a-t-elle été appelée à collaborer au Concours international de composition à Shanghai et comment a-t-elle procédé à la sélection des huit compo-

4. Pour en connaître davantage sur Walter Boudreau: www.smcq.qc.ca/smcq/fr/artistes/b/boudreau_wa/ (consulté le 19 octobre 2017).

5. Pour en connaître davantage sur José Evangelista : www.smcq.qc.ca/smcq/fr/artistes/e/evangelista_jo/ (consulté le 19 octobre 2017).

6. Pour en connaître davantage sur Denis Gougeon : www.musique.umontreal.ca/personnel/gougeon_d.html (consulté le 19 octobre 2017).

7. Pour en connaître davantage sur Simon Bertrand: www.simbermusique.com/ (consulté le 19 octobre 2017).

8. Pour en connaître davantage sur Pierre Michaud: www.pierremichaud.info/ (consulté le 19 octobre 2017).

9. Pour en connaître davantage sur Analía Llugdar: www.analiallugdar.net/ (consulté le 19 octobre 2017). sitrices et compositeurs qui ont participé à ce concours? Quels ont été les prémisses ou les critères qui vous ont guidés dans la sélection de ceux-ci?

Walter Boudreau : C'est un concours de circonstances malheureuses qui s'est retourné en notre faveur: à l'époque, René Bosc et moi avions planifié que la tenue de la version 2009 de Présences, le grand festival de Radio-France, aurait lieu à Montréal. C'était un projet énorme et formidable! Mais entre-temps, des changements sont survenus au niveau de la direction de Radio-France et le nouveau directeur général nous a laissé tomber dans ce projet en nous menant vers un cul-de-sac. Alors, j’ai demandé à la présidente de notre conseil d'administration d'écrire au président de Radio-France pour lui dire qu'on nous avait menés en bateau et donc, que nous nous attendions à une compensation quelconque. De fait, nous avons eu droit à plusieurs compensations et l'une d'elles a été d'inclure huit compositrices et compositeurs québécois dans le projet du concours de composition à Shanghai. C'est donc un des "prix de consolation" que nous avons eus pour compenser le fait que la tenue du Festival Présences n'aurait pas lieu à Montréal dans le contexte du Festival Montréal/Nouvelles Musiques (MNM) cette année-là.

Ainsi, je suis devenu le mentor de nos participants québécois et c'est moi-même qui les ai choisis. Première des choses, c'était de trouver des compositrices et compositeurs qui ont un style très différent les uns des autres, mais aussi une approche personnelle et une feuille de route remarquable. Or il fallait que ces personnes aient l'esprit assez ouvert pour se plier à ce jeu-là, aux paramètres de la commande (écrire 
une œuvre pour instruments traditionnels chinois, imposition d'une mélodie traditionnelle chinoise ${ }^{10}$, etc.) J'ai donc approché des gens que je croyais être en mesure de répondre favorablement et de façon très professionnelle à la commande. Les huit sélectionnés étaient vraiment une brochette représentative, tant au niveau de l'âge que de la diversité des styles, et cela a été très intéressant de voir comment tout ce monde-là s'est distingué dans cette aventure.

À titre de principal organisateur de la présence québécoise au sein de l'évènement, comment s'est déroulée votre expérience? Avez-vous rencontré des contraintes lors de l'organisation de ce projet d'envergure? Le cas échéant, comment les avez-vous surmontées?

Walter Boudreau: Nous avons fait ça comme des pros! Bien sûr, il y a eu des obstacles institutionnels, mais nous les avons surmontés. La plus grosse difficulté était d'aller chercher les subventions: il fallait trouver les fonds nécessaires pour commander les œuvres qui allaient être créées et pour se rendre à Shanghai. Nous avons donc fait appel au Conseil des arts et des lettres du Québec (CALQ) et au Conseil des arts du Canada (CAC). Mais une fois arrivés en Chine, nous avons été pris en charge par les responsables du concours. Et nous avons été incroyablement bien reçus et très impressionnés par la ville de Shanghai.

10. Des mélodies chinoises étaient imposées pour le concours et la SMCQ a fourni aux compositrices et aux compositeurs les enregistrements nécessaires, chacun ayant reçu son propre $C D$.

\section{Préparatifs}

Quel rapport entreteniez-vous avec les concours de composition avant votre participation au Concours international de composition à Shanghai? Aviez-vous déjà participé à d'autres concours ou aviez-vous tendance à fuir ce genre d'évènements?

Denis Gougeon : J'avais déjà participé à des concours avant. Forcément, je crois que j'entretiens un rapport sympathique et positif avec les concours.

Simon Bertrand: Avant Shanghai, je n'avais jamais participé à un concours d'une telle envergure. On peut dire que Shanghai est mon premier grand concours en tant que compositeur, mais il faut mentionner ici que j’ai commencé la composition assez tardivement dans ma carrière de musicien.

Pierre Michaud: Je n'affectionne pas particulièrement les concours qui s'inscrivent dans une discipline artistique, car l'opinion que l'on reçoit est restreinte au jugement des membres du jury. Mais lorsqu'il y a un prix du public, c'est différent. Toutefois, il faut se forcer à en faire, car ils permettent d'acquérir des subventions et d'obtenir le jugement des pairs.

Analía Llugdar: J'avais participé à plusieurs concours canadiens avant Shanghai. Entre autres, j’ai remporté le prix Jules-Léger du CAC, le prix Québec-Flandre de la SMCQ et du CALQ, le premier prix du Concours national des jeunes compositeurs CBC/Radio-Canada en 2003, etc. Les concours de composition constituent indubitablement une opportunité unique, notamment pour les jeunes compositrices et compositeurs, de faire connaître leur musique et d'être joués par des musiciens et des ensembles professionnels. 
Comment avez-vous été contactés par la SMCQ pour participer à ce concours international de composition et quelles ont été vos réactions?

Denis Gougeon : J'ai accepté cette aventure-là. D'autant plus que les contraintes m'intéressaient: aller à la rencontre d'une culture qui n'est pas la nôtre, découvrir leur folklore et leurs traditions musicales... Bref, le défi était là. Mais aussi, ce qui était excitant là-dedans, c'est qu'on était une "gang de chums"!

Simon Bertrand : Je vivais au Danemark à ce momentlà. C'est Walter Boudreau qui m’a appelé pour m'annoncer que j’avais été sélectionné pour participer à Shanghai, probablement parce que j'avais déjà une expérience avec la culture japonaise et les instruments orientaux.

Analía Llugdar: J'ai été avant tout séduite par l'idée de découvrir la musique et la culture chinoises, qui étaient à ce moment-là étrangères à moi. J'étais ravie d'avoir le privilège de faire partie du groupe de compositrices et compositeurs choisis pour représenter le Canada lors de ce concours de si grande envergure.

Quelles sont les prémisses qui vous ont guidé(e) dans l'approche du Concours international de composition à Shanghai et dans l'ouvre à concevoir? Par exemple, la perspective de remporter ce concours vous a-t-elle influencé(e) dans la conception de l'œuvre?

José Evangelista: J'ai préféré me concentrer sur la composition en elle-même plutôt que sur la possibilité de gagner le concours.

Simon Bertrand: Lors de mon premier séjour, je suis resté trois ou quatre jours de plus après le départ de mes collègues. J'étais donc toujours en Chine au moment où s'est produit le séisme de 2008 au Sichuan ${ }^{11}$. Cet évènement a grandement influencé mon œuvre (que j’ai dédiée aux victimes) et le choix de la mélodie traditionnelle. J'ai repris une mélodie plutôt joyeuse du Sichuan et je l'ai ralentie de manière extrême pour en faire une espèce de chant mortuaire.

Pierre Michaud: Comme je ne suis pas un "fan" des concours, je ne voulais pas écrire une pièce de concours, mais bien une œuvre qui allait plaire aux auditeurs ainsi qu'à moi-même. Par contre, j'ai été un peu déconcerté d'apprendre, seulement une fois arrivé sur place, que des thèmes étaient imposés.

Analía Llugdar: Une mélodie nous avait été imposée et nous devions la respecter. La seule prémisse pour moi était donc de créer une œuvre [In the Rays of the Oriental Pearl] qui puisse fusionner la couleur des erhus ${ }^{12}$, l'instrument de mon choix, aux instruments occidentaux de l'orchestre. J'ai voulu mettre en valeur la force de la culture musicale chinoise que j’allais découvrir là-bas.

\section{Démarches et processus de création}

Quels sont les intérêts qui vous ont guidé(e) lors de votre premier voyage à Shanghai et comment la rencontre de la culture chinoise a-t-elle été une source d'inspiration?

José Evangelista: J'ai voulu connaître de l'intérieur une culture qui m'intéresse beaucoup.

11. Le séisme est survenu le 12 mai 2008 et a causé des ravages d'une grande ampleur dans la province chinoise.

12. Le erhu est un instrument qui appartient à la famille des huqins (vielles chinoises). Il rappelle le violon occidental, mais n'a que deux cordes. 
Denis Gougeon: On était là dans un but d'apprentissage et non pas de compétition. D'ailleurs, on ne pouvait pas décider de plaire à l'avance, car nous ne savions pas qui étaient les membres du jury et encore moins leurs intérêts musicaux. Et il y avait aussi un vote du public dont nous ignorions le poids. II y a donc eu trois chocs: le choc culturel, celui de l'écoute du dizili3 et le choc de la mélodie Paysage de Wuxi ${ }^{14}$. Cela m'a inspiré dans le choix de faire une sorte de roman sonore pour l'oreille ${ }^{15}$

Simon Bertrand: Ma pièce s'intitule Duels. II s'agit autant du duel entre l'homme et la nature (en référence au séisme de Sichuan) que de celui entre deux solistes, le Konzertmeister de l'orchestre et un erhu. Mais ce dernier duel est une sorte de fusion dans la rencontre des deux cultures propres aux deux instruments. En hommage aux victimes du séisme, mon œuvre est sous-titrée "Carrying the red lanterns" en référence à un rituel de protection traditionnel chinois, un trompe-l'œil où il s'agit pour les villageois d'allumer des lanternes rouges afin de faire croire aux dieux que leur village est en feu pour que ne s'abatte pas sur lui leur colère.

13. Le dizi est une flûte traversière chinoise faite de bambou. Bien que son corps soit généralement percé de 12 trous et recouvert d'une membrane de roseau, le dizi choisi par Denis Gougeon possédait sept trous et le dernier était couvert d'une membrane appelée dimo.

14. On peut entendre cette mélodie traditionnelle ici: www.youtube.com/watch?v=qBblQ_dSYok (consulté le 11 septembre 2017).

15. Dans le numéro que Circuit a consacré à l'œuvre de Denis Gougeon, François-Hugues Leclair se penche sur la dimension narrative de l'œuvre de Gougeon et analyse Toy (Music Box) dans cette perspective. Voir : François-Hugues Leclair (2014), "Denis Gougeon: entre affect et narrativité", Circuit, musiques contemporaines, vol. 24, $\mathrm{n}^{\circ}$ 1, p. 51-65.
Pierre Michaud : Je suis allé à Shanghai avec une enregistreuse pour capter le plus d'éléments possible. Ma pièce, Carnet de voyage, expose les impressions que la Chine m'a laissées, des sons de l'avion aux paysages et aux mentalités chinoises. La Chine est pourvue d'une culture impérialiste. C'est aussi un pays de contradictions, de paradoxes: par exemple, au niveau de l'architecture, nous croisons tout autant de vieux bâtiments érigés dans une esthétique traditionnelle que d'autres dans une esthétique urbaine et plus contemporaine. Or cela a été un séjour plutôt court et très contrôlé. Nous n'avions que très peu de liberté dans nos temps libres. II y a donc eu une certaine contrainte au niveau du temps.

Analía Llugdar: Lors de ce premier voyage, mon intérêt principal était de découvrir la sonorité des instruments traditionnels chinois, pour ensuite imaginer leur amalgame au niveau des timbres avec les instruments occidentaux. Deux évènements ont été marquants et décisifs pour moi. Premièrement, la rencontre avec l'Orchestre national d'instruments traditionnels de Chine, composé dans son intégralité d'instruments traditionnels, avec leur sonorité totalement inouïe et fascinante. Le second évènement a été la rencontre avec la directrice du Conservatoire de musique de Shanghai, une dame très âgée, qui nous a parlé de la musique traditionnelle chinoise tout en racontant l'histoire de certaines des mélodies qui nous étaient imposées pour le concours. Ces rencontres ont dévoilé ce qui m'est apparu être le cœur de cette musique: la notion du "temps", différente de celle que je concevais jusqu'alors, le souci de la ligne mélodique comme conductrice de la forme musicale, le recours aux sonori- 
tés brillantes, vives et parfois criantes de certains instruments, les rythmes caractéristiques et, finalement, les multiples timbres des percussions traditionnelles. J'ai donc cherché à travers cette démarche à transmettre la force expressive de cette culture qui m’a captivée.

Qu'est-ce qui a motivé votre choix pour la sélection d'instrument(s) traditionnel(s) chinois et comment avezvous procédé à leur fusion avec l'orchestre occidental?

José Evangelista : J'ai voulu m'instruire sur le guqin, un instrument que j'admire.

Denis Gougeon: J'ai choisi le dizi pour la sonorité de bambou et parce que c'est probablement le plus vieil instrument qui existe (après les percussions!). Pour moi, c'était le souffle, le chant qui s'apparente au Paysage de Wuxi. Et cette mélodie m’a profondément touché, intéressé. Simple, mais efficace et très belle, comme une jolie berceuse où l'harmonisation m'offrait une liberté amusante. Le souffle est une notion importante pour moi. Le dizi, c'est un peu comme le shakuhachi, une flûte droite ${ }^{16}$, mais qui est intimement lié au souffle de l'interprète. Le souffle, c'est aussi l'âme de l'interprète. Le dizi étant un instrument diatonique, la possibilité du chromatisme y est assez difficile. Alors, j’ai eu l'idée d'écrire pour deux dizis et ainsi, je pouvais partager le chromatisme entre eux. En ce qui concerne l'alliage entre ces deux cultures, cela ne m'a pas posé de problème. C'est un peu comme la cuisine: on invite un bon chef à concocter un plat avec des ingrédients chinois et occidentaux. Si je tenais à rester fidèle au folklore, à la mélodie initiale, je lui ai toutefois donné un tout autre contexte, un autre espace culturel [voir Figure 2].

16. Il s'agit d'une flûte qui se joue à la verticale.
FIGURE 2 Toy (Music Box) de Denis Gougeon: page huit de la partie de dizi 1.
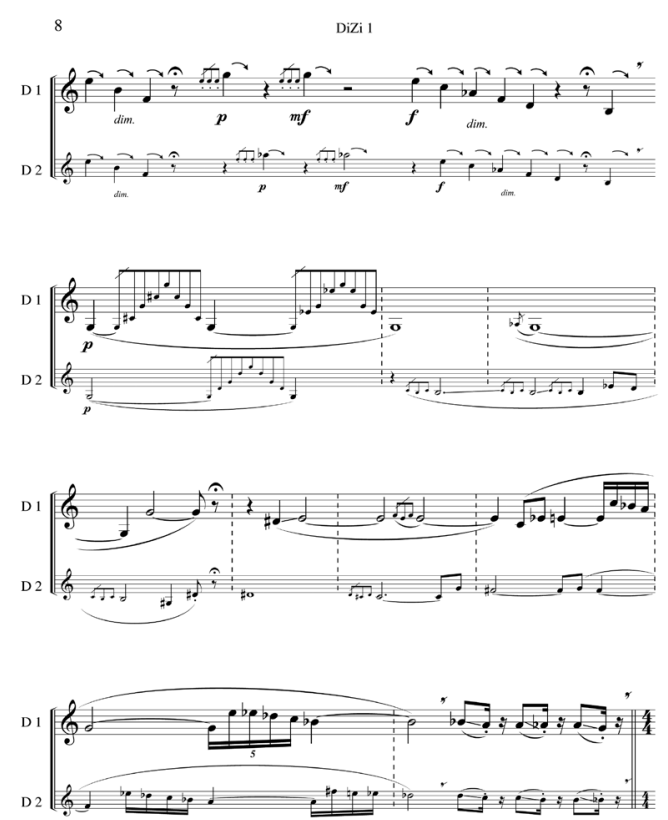

Simon Bertrand: J'ai choisi l'erhu, proche cousin du violon occidental, pour sa sonorité unique qui me rappelle la voix humaine.

Pierre Michaud: Nous avions été conviés à passer une journée au Conservatoire de musique de Shanghai au cours de laquelle des musicologues et des interprètes nous ont présenté différents instruments traditionnels et différentes musiques issues du folklore. Les interprètes ont grandement influencé le choix de l'instrument: c'est l'enjeu humain de l'interprétation. Je dois avoir envie de travailler avec telle personne ou telle autre et c'est un facteur qui a grandement influé sur mon choix des instruments. 
Analía Llugdar : J'ai choisi de travailler avec deux erhus et deux zonghus ${ }^{17}$, car leurs sonorités me renvoyaient de manière spontanée à la richesse et à la multiplicité des couleurs et des contrastes que j'avais perçus tant dans la musique, dans la culture que dans la ville de Shanghai. J'ai travaillé l'écriture de ce quatuor de solistes comme s'il s'agissait d'un seul instrument, avec une texture homogène, mais avec des voix multiples qui s'imitent, se dédoublent et résonnent dans un contrepoint qui, lui, finira par s'étendre à tout l'orchestre. La structure de la pièce développe l'idée de contraste qui s'exprime dans l'œuvre par la confrontation entre des moments lyriques et d'autres plus percussifs et agressifs.

Comment innover et s'exprimer personnellement dans le contexte d'une ouvre de cing minutes, notamment dans le cadre d'un concours musical?

Walter Boudreau: C'était une partie du défi à relever. Chaque compositeur savait que ces cinq minutes constituaient le germe de ce qui était pour en durer environ quinze. D'une certaine manière, chaque compositeur faisait sa propre réduction. Généralement, les restrictions de durée permettent d'offrir la chance à tout le monde d'être entendu: tu as cinq minutes pour nous montrer de quel bois tu te chauffes! Selon moi, tout le monde a bien relevé le défi. Un des grands exemples que nous pouvons trouver de ce côté, c'est Anton Webern, n'est-ce pas?

Denis Gougeon : J'ai construit l'œuvre sur l'image de deux personnes qui contemplent un paysage et le com-

17. Cet instrument est une version plus grande de l'erhu. Il possède également deux cordes, mais sa sonorité est légèrement plus grave que celle de son analogue. mentent en se remémorant des choses. Ici, Paysages de Wuxi agit comme le fil conducteur de ces souvenirs-là. Plus généralement, il faut construire une forme musicale qui soit la plus adaptée et la plus adéquate en fonction du discours voulu. J'ai laissé la finale sur un point d'exclamation ou trois points de suspension, comme si une suite était possible. Cela m’a permis de répondre au critère de la durée. Une œuvre de cinq minutes semble toujours une contrainte dans le milieu. Pour ma part, cela ne me pose pas de problème. Lorsque je sais que j'ai une limite de temps, je moule mon idée en fonction de cela.

Simon Bertrand: J'avais déjà préconçu la pièce en entier sous la forme de deux mouvements. J'ai trouvé la contrainte du temps très pénible: la durée était trop courte! Cela a même constitué un problème majeur dans mon processus d'écriture. À peine l'idée musicale commençait-elle à être perceptible dans l'œuvre que je me rendais compte que la pièce était déjà d'une durée de trois à quatre minutes! Duels est donc une œuvre d'une quinzaine de minutes compressée en sept minutes. Elle était peut-être trop dense.

Pierre Michaud: II m'est apparu difficile de travailler avec du matériel imposé [instrument et mélodie] : c'est complexe de reprendre ces matériaux qui sont lourds du côté des connotations qu'ils portent. De notre point de vue occidental, on se demande si on reprend bien la mélodie; on ne veut pas trop la dénaturer et lui faire perdre tout son sens.

Analía Llugdar: Ce n'est pas le désir de l'innovation qui me préoccupait, mais plutôt la volonté de me laisser porter par l'influence de cette musique fascinante et débordante d'expressivité. 
Comment se sont déroulées les répétitions menant à la création de l'ouvre et quelles sont les contraintes rencontrées?

Walter Boudreau : La première année, nous avions eu un très bon chef. Or l'année suivante, c'était un autre chef et il ne semblait avoir ni l'ouverture d'esprit nécessaire ni l'expérience de son prédécesseur. Mais le travail s'est déroulé de manière très professionnelle. II faut dire que je ne le connaissais pas dans un autre répertoire, mais dans le répertoire qu'il avait à défendre. Et je ne le sentais pas à son meilleur : il semblait avoir une grande difficulté dans la compréhension des partitions. Le chef en comprenait les signes, les symboles, mais pas le sens... C'est comme s'il lisait une série de mots sans les mettre en relation.

José Evangelista : Elles se sont déroulées de manière très harmonieuse: chaque candidat s'intéressait au travail des autres.

Simon Bertrand: Les répétitions se sont, dans l'ensemble, bien déroulées. Cependant, il y a eu quelques contraintes imputables à la barrière érigée par la langue. Le chef d'orchestre ne parlant pas le français et très peu l'anglais, nous avons été dans l'obligation d'avoir recours à un intermédiaire qui assurait le rôle de traducteur. Le chef semblait sous pression (ce qui était justifié !), mais cela ne faisait que rendre les communications plus difficiles. Il est essentiel qu'un compositeur et un chef d'orchestre communiquent et travaillent de manière solidaire. II y a donc eu quelques accros du côté de la communication puisque le chef n'était pas très disposé à nous écouter, ce qui a été vexant pour moi et d'autres compositeurs. Mécontents, nous avons demandé à l'assistant chef d'établir une rencontre afin que nous puissions régler ce problème de communication. Après cette rencontre, tout s'est placé!

Analía Llugdar: Les répétitions se sont plutôt bien déroulées: les musiciens étaient très ouverts et nous avons échangé beaucoup par rapport à la notation, à la sonorité désirée, aux modes de jeux, etc. Seul bémol, quelques désaccords "techniques" avec le chef d'orchestre...

\section{Résultats et bilan}

Quelles ont été vos réactions suite à l'annonce des résultats?

Walter Boudreau : Ceux qui ont été retenus étaient très contents. Ceux qui n'ont pas été retenus, c'est comme dans n'importe quel concours: il y a des aléas qui viennent avec les concours et c'est comme jouer à pile ou face. C'est qu'il y a une question de hasard là-dedans. Le public votait. Et j’avais moi-même fait un vote, dans ma tête, par rapport à la façon dont le public allait voter. Alors j'étais très content, surtout qu'il s'est formé de belles amitiés dans cette aventure! Par contre, je n'étais malheureusement pas là lorsque Denis a gagné la grande finale en 2010. Normalement, j'aurais dû l'accompagner, mais j'étais à la Triennale de Cologne à ce moment-là. Par contre, j'étais très fier et content lorsque j'ai reçu un courriel de lui dans lequel il m’annonçait sa victoire. J'étais là, mais en pensée!

José Evangelista: Une réaction normale, comme lors d'une épreuve sportive.

Denis Gougeon : J'étais très heureux et satisfait. Mais je n’ai aucune idée de ce qui a pu favoriser le fait que j’aie 
gagné: est-ce parce que la mélodie folklorique [Paysage de Wuxi] était facilement et clairement reconnaissable, est-ce l'esthétisme, est-ce le thème, etc.? II m'est impossible de définir ce qui a penché en ma faveur et c'est pourquoi je ne me vante pas de cette victoire...

Simon Bertrand: Bien que je souhaitais évidemment gagner un des prix pour des raisons purement pécuniaires, je me doutais d'avance en même temps que je ne gagnerais pas à cause du sujet dont traitait ma pièce et de son expression un peu morbide (par la mélodie joyeuse de Sichuan qui s'est transformée en requiem). Selon moi, cette manière de faire n'est pas une pratique qui concorde avec la culture asiatique. Je tenais toutefois à rester le plus pur et le plus authentique possible par rapport à mon idée musicale. Cependant, Walter Boudreau m'a apporté un réconfort artistique en me confiant qu'il avait eu des frissons lors de la création de Duels. Cela a été le début d'une grande collaboration entre lui et moi.

Pierre Michaud: Je n’ai pas de regret. La règle première Iorsqu'on participe à des concours, c'est de ne pas avoir d'attente, puisque la décision finale est remise entre les mains d'un jury composé de seulement quelques individus. À mon avis, toutes les œuvres qui ont été créées étaient des œuvres de grande qualité.

Considérez-vous que votre participation au Concours international de composition à Shanghai a été fructueuse? Quelles seraient les modifications que vous apporteriez si une participation de ce niveau se représentait?

Walter Boudreau: Oui, ç'a été très fructueux! D'abord, cela a permis à huit de nos compositrices et compositeurs d'être joués à Shanghai. Ensuite, nous y avons tellement appris: les compositrices et compositeurs avaient une belle propension à découvrir le canon musical de la Chine. Nous sommes revenus de là enrichis musicalement. Je dirais sans hésiter que l'expérience a été gagnante pour tous. Même pour ceux qui n'ont pas gagné. Par exemple, la pièce de Sean Pepperall a été reprise là-bas. Par contre, ce que je déplore, c'est le manque de moyens pour renforcer, entretenir et poursuivre les liens que nous avons tissés à Shanghai. Nous avons manqué de moyens pour donner une suite immédiate à tout cela: cette aventure n'a pas été un one night stand, mais pas loin... Continuer à collaborer avec ces gens que nous avons côtoyés et renforcer nos rapports avec eux, ces défis relèvent plus de la complicité que de la business. Mais ce genre de collaboration, ça prend une vision à long terme et pour l'instant, il n'y a pas de plan précis... Je n'ai pas eu les moyens financiers de poursuivre cette aventure comme je l'aurais voulu, mais je garde en tête qu'il faut trouver des moyens de renvoyer l'ascenseur, ce qui pourra peutêtre advenir un jour.

José Evangelista : Très fructueuse, une expérience stimulante. Je ne toucherais pas au format proposé par le Concours international de composition à Shanghai. De plus, écrire pour des instruments traditionnels chinois est une expérience considérable à titre de compositeur occidental.

Denis Gougeon : J'ai beaucoup appris en étant en contact avec cette culture que je connaissais peu : cela m'a ouvert l'esprit et les horizons. J'ai gagné une reconnaissance aussi. 
Simon Bertrand : Je n'apporterais pas de modification, même si je n'ai jamais été enclin à composer dans le but de plaire à un jury, ou pour gagner un concours. Et je le suis encore moins aujourd'hui! Toujours rester authentique, être dans l'idée d'exprimer quelque chose d'une manière forte à autrui. Duels a été une œuvre "tremplin" dans mon répertoire et c'est elle qui a convaincu l'Orchestre symphonique de Longueuil de me prendre comme compositeur en résidence.

Pierre Michaud: Je garde un souvenir très positif de cette expérience. Pour nous, le contexte était très amical et l'aspect compétitif peu décelable. Un concours artistique ne devrait jamais prendre la forme d'une compétition sportive. Personnellement, si on me donne le choix entre participer à un festival ou un concours à l'étranger, je choisis sans conteste le festival. Par contre, j'ai moins aimé l'aspect de contrôle: il y avait trop d'organisation et de restrictions. Notre horaire était prédéterminé à l'avance et nous étions toujours ensemble. Même les activités touristiques avaient été organisées: je n'ai pas pu voir la Chine, mais une Chine, soit celle que nous devions découvrir selon les organisateurs...

\section{Postlude}

En quoi de tels concours sont-ils importants pour soutenir et développer la carrière de compositeur, et plus généralement, la création musicale?

Walter Boudreau : D’abord, nul n'est prophète en son pays. Les grandes carrières se font - sauf exception - à l'international. La géographie de la musique contemporaine est constituée d'une série de petits îlots dispersés un peu partout sur la planète. C'est capital de continuer à développer ce réseau-là. En ce sens, les concours sont une aide précieuse: en plus de faire circuler les idées et la musique, ils procurent un revenu et un nom à l'étranger tout en diversifiant les sources de financement pour les compositrices et compositeurs.

José Evangelista : Dans le cas du Concours international de composition à Shanghai, l'opportunité d'avoir eu un orchestre à notre disposition ainsi que des rencontres avec des musicologues chinois, est un élément considérable dans le développement d'une carrière de compositeur.

Denis Gougeon : L'acquisition de connaissances et d'expériences est plus importante que le prix en soi. Du moins, c'est ce que je retiens de mon expérience à Shanghai. Cette expérience surpasse même le fait d'avoir écrit une pièce pour orchestre! De plus, cette expérience me sert encore aujourd'hui, particulièrement du côté de la musique japonaise que j’aime étudier et comparer, tant avec la musique chinoise, que je connaissais moins, que celle occidentale ${ }^{18}$.

Simon Bertrand: Ici, je ne peux que répondre avec un scepticisme global face à l'utilité réelle et concrète qu'ont de tels concours. Qu'une œuvre ait gagné un concours, c'est bien, mais encore faut-il qu'un chef d'orchestre et des musiciens aient envie de la rejouer ! Et cette réalité n'est pas forcément en corrélation avec le fait de remporter des concours. Par contre, financièrement, c'est certain que les concours peuvent aider les compositrices et compositeurs. De mon côté, je suis

18. Toy (Music Box) a été créée en première nord-américaine par l'Orchestre symphonique de Montréal (OSM) le 26 février 2014 dans le cadre de la Série hommage 2013-2014 de la SMCQ, laquelle était consacrée à Denis Gougeon. 
FIGURE 3 Denis Gougeon en compagnie des deux solistes chinois, Qian Jun et Jin Kai, qui ont créé Toy (Music Box), lors d'une répétition à Shanghai en mai 2010. Photo : Marie-Danielle Parent.

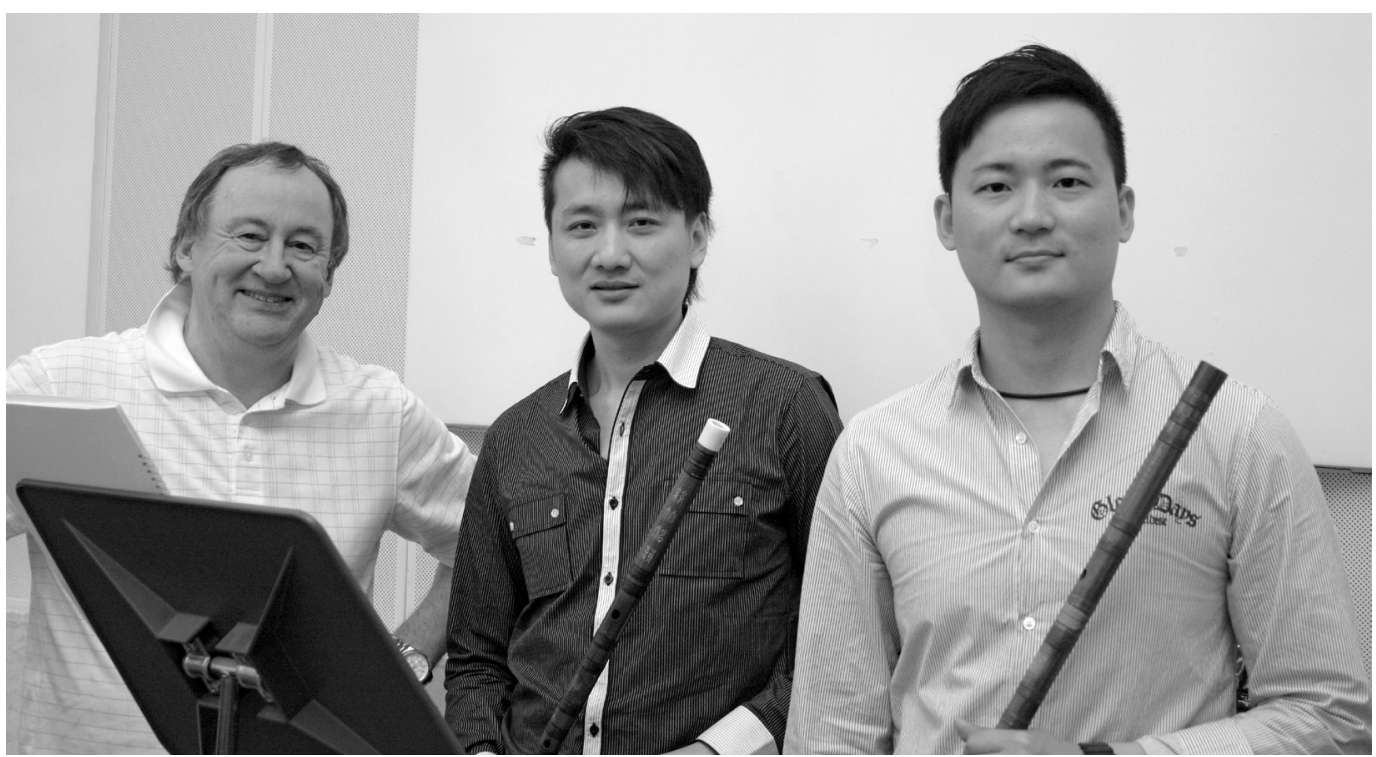

plutôt en faveur des prix qui vont récompenser l'ensemble de l'œuvre d'une compositrice ou d'un compositeur, par exemple le prix Opus dans cette catégorie ${ }^{19}$. Je crois que la qualité la plus importante dans ce métier, c'est la résilience. L'œuvre d'un compositeur commence avec sa première œuvre et se termine par sa dernière, nonobstant ses succès ou ses échecs dans ce genre de concours ou dans sa carrière.

Pierre Michaud: Remporter un concours aide certainement à la carrière d'une compositrice ou d'un compositeur. C'est quelque chose qui se met bien dans un

19. Lors de son gala annuel intitulé les prix Opus, le Conseil québécois de la musique (CQM) remet le prix "Compositeur de l'année" assorti d'un montant de 10000 \$ offert par le CALQ. curriculum vitce. Et puis, lorsqu'on est professeur, nous avons la responsabilité de rayonner et d'assurer une certaine visibilité de l'établissement au sein duquel nous enseignons. Par contre, en ce qui concerne la création musicale en elle-même, la participation à un concours n'apporte pas réellement quelque chose. J'irais même jusqu'à dire que les concours peuvent nuire à la création musicale. C'est qu'à mon avis, les contextes compétitifs ne favorisent pas la création. D'un autre côté, un tel contexte peut attirer un public nouveau qui, sans cela, n'aurait probablement jamais assisté à un concert de musique contemporaine.

Analía Llugdar: Un concours avec de telles caractéristiques permet à la compositrice et au compositeur de 
découvrir une autre culture et une autre manière de penser la musique. Cette remise en question et cette confrontation culturelle ont une influence indéniable sur le faire musical ainsi que sur la manière de penser les sons, les durées, les gestes, la forme, etc. II s'agit d'occasions privilégiées pour nous faire rompre avec des présupposés, bref l'opportunité de reconstruire, à nouveau, à partir de matériaux et de chemins qui nous étaient auparavant inconnus.

\section{De la nécessité des concours de composition}

Il va de soi qu'un concours de cette ampleur pourrait faire l'objet d'études plus poussées sur plusieurs plans (analyse des œuvres, retombées professionnelles, etc.) et, en ce sens, les témoignages recueillis ici peuvent s'avérer d'une grande utilité. Les horizons que des- sinent les propos des acteurs à la base de cette enquête sont multiples et profondément variés, faisant ainsi la démonstration que leur participation au Festival Présences à Shanghai a laissé une empreinte durable, toujours présente plus de 10 ans après leur arrivée dans la ville chinoise. Si certaines des impressions recueillies sont presque unanimement partagées, d'autres, en revanche, font preuve d'une dimension plus intime. Voilà qui contraste avec la sphère professionnelle qui, lorsque vient le temps de rendre compte d'expériences du genre, est plus souvent mise de l'avant par rapport aux aspects plus informels et dont les répercussions peuvent s'avérer hautement signifiantes, comme les liens tissés avec les interprètes chinois, le plaisir de découvrir la musique traditionnelle chinoise ou la satisfaction personnelle d'avoir relevé le défi du concours et de l'acculturation. 